\title{
Proceeding
}

Supplementary Issue: Autumn Conferences of Sports Science. Costa Blanca Sports Science Events, 18-19 December 2020. Alicante, Spain.

\section{Moving through didactic of human movement and bodily experience: A motion analysis preliminary study}

\author{
VALERIA AGOSTI $1 \square$, SILVIA COPPOLA², RODOLFO VASTOLA ${ }^{2}$ \\ ${ }^{1}$ Department of Human and Social Sciences, University of Bergamo, Italy \\ ${ }^{2}$ Department of Human, Philosophical and Educational Sciences, University of Salerno, Italy
}

\begin{abstract}
Dance is considered an educational-pedagogical strategy useful in motor learning for improving mobility in the elderly. Ageing people are often involved in falling episodes that occur during daily activities, such as walking. The purpose of this pilot study was to compare two different didactic approach on gait parameters of elderly: Dance and aerobic physical activity. By means of a motion analysis approach, 20 ageing people without cognitive, neurological or orthopaedic problems were enrolled. Data were collected using an optoelectronic system (BTS $\mathbb{B}$ ). Participants were randomly assigned to Dance (Dg) or Physical activity (PAg) groups. Gait parameters were evaluated at study entry (t0) and at 10 weeks ( $\mathrm{t} 1$, end of training). Statistical analysis was performed using repeated measure ANOVA. Results showed that at to the two groups did not differ for all parameters. At t1 a significant improvement in gait parameters was observed in Dg group. Within $\mathrm{Dg}$, post hoc comparison between to and $\mathrm{t} 1$ showed a significant effect for several gait parameters. Within $\mathrm{PAg}$, no significant differences were found in all examined parameters. In conclusion, our preliminary findings showed that Dance is a bodily experience that may target primary prevention for ageing falls and benefit motor learning and locomotion.
\end{abstract}

Keywords: Education; Didactic; Movement; Learning; Elderly; Motion analysis.

\section{Cite this article as:}

Agosti, V., Coppola, S., \& Vastola, R. (2021). Moving through didactic of human movement and bodily experience: A motion analysis preliminary study. Journal of Human Sport and Exercise, 16(2proc), S574-S579. doi:https://doi.org/10.14198/ihse.2021.16.Proc2.41

Corresponding author. Department of Human and Social Sciences, University of Bergamo, Piazzale S. Agostino 2 - 24129 Bergamo (BG), Italy. https://orcid.org/0000-0002-1237-7097

E-mail: valeria.agosti@unibg.it

Abstract submitted to: Autumn Conferences of Sports Science. Costa Blanca Sports Science Events, 18-19 December 2020. Alicante, Spain.

JOURNAL OF HUMAN SPORT \& EXERCISE ISSN 1988-5202

(c) Faculty of Education. University of Alicante

doi:10.14198/jhse.2021.16.Proc2.41 


\section{INTRODUCTION}

Falls in the elderly are a huge problem for public health, much debated in the scientific literature, as is the study of strategies useful for their primary prevention. It has been shown that falls in the elderly are often attributable to Activity Daily Living (ADL), particularly during walking (Cruz-Jimenez, 2017). It has also been shown that physical performance, especially aerobic activity, can contribute to improve walking parameters in the elderly and consequently could be effective as a primary prevention factor for falls. However, the right "recipe" for physical activity has not been found yet (Faber et al., 2006; de Labra et al., 2015), so which is the right physical activity? Physical activity interventions, not only for the elderly, are often not very effective both in terms of physical efficiency and in educational terms (Hui \& Rubenstein, 2006). The explanation for this failure could lie not so much in the type of activity but rather in the didactic modality proposed. Moreover, physical activity interventions on the elderly are hardly conducted with an educational purpose or paying attention to the setting and use of communication channels that tend to break down the hesitations that the elderly themselves have in participating in physical activity.

Evidence has now emerged about the need to adopt pedagogical and didactic strategies in physical activity interventions for the elderly in order to lead them towards a real change in motor habits. Pedagogy is both empirical and practical science (Stein, 2017), didactics is the theory aimed at the teaching and learning contents, which are the educational goals. With a purely pedagogical orientation, the educational goals in the didactic proposal of physical activities for the elderly must have a tailored personalization of the programs. The elderly has very different needs from young people or adults and proposing activities, environments and teaching methods suitable for them is the new challenge in the educational research of motor activities (Brawley, 2003). Dance, similar to aerobic physical activity in its classification of metabolic performance, seems to have beneficial effects for the elderly, and not only on motor but also on cognitive and social parameters (Veronese et al., 2017; Rodriguez et al., 2019).

In fact, Dance differs from other physical activities because it represents an enriched motor experience (Dhami et al., 2015) and for this reason, Dance interventions involve multiple functions and are intrinsically multimodal. In fact, dancing does not only mean the movement of the body, but also involve the levels of learning, attention, memory, emotion, rhythmic coordination, balance, visuospatial capacity, acoustic stimulation, imagination, improvisation and social interaction (Mattle et al., 2020). For these reasons, in structuring the activity programs, Dance teachers must always think about using teaching strategies that make it possible for everyone, including the elderly, to learn very complex movement patterns. Many studies investigated the relationship between Dance activities and the prevention of falls in the elderly (Veronese et al., 2017; Rodriguez et al., 2019) but to our knowledge few studies studied the direct effects of Dance on the change of walking parameters in the elderly, none using an optoelectronic motion analysis system.

The aim of this work is to evaluate, through the instrumental measurement of the spatiotemporal parameters of the gait cycle, if and what differences in the organization of motor function may be consequent to two different aerobic exercise interventions for the elderly. Both interventions require a similar metabolic work but there are big differences both in the involvement of the body districts and in the organization of teaching in the gym. The findings, strong points, limitations and scope of future research in this field will be discussed.

\section{MATERIAL AND METHODS}

20 elderly subjects, 10 men and 10 women participated in the study (see Table 1 for demographic data). All subjects had no ongoing and no history of neurological or orthopaedic pathologies that could interfere with 
motor performance. None of them had experience in Dance or any other physical activity. The University Research Ethics Committee approved the project and signed informed consent was obtained for all participants. Data were collected at the Motion Analysis Laboratory at University of Salerno (Italy).

Table 1. Demographic data of participants.

\begin{tabular}{lcc}
\hline & Dance group $(\mathrm{Dg})$ & Physical Activity group (PAg) \\
\hline Age $(\mathrm{yrs})$. & $73 \pm 2.708$ & $74.4 \pm 2.270$ \\
Sex $(\mathrm{m} / \mathrm{f})$ & $5 / 5$ & $5 / 5$ \\
Weight $(\mathrm{kg})$ & $72.9 \pm 8.987$ & $73.4 \pm 8.90$ \\
$\mathrm{H}(\mathrm{cm})$ & $167.9 \pm 10.535$ & $167.8 \pm 10.411$ \\
\hline
\end{tabular}

Note: yrs.: years; m: male; ; female; kg: kilograms; $H$ : height; cm: centimetres.

Participating subjects was asked to walk along a 10-meter walkway at a comfortable pace for their personal gait. For the detection of the main spatiotemporal components of the gait cycle, an optoelectronic system $(\mathrm{BTS} \otimes)$ at $120 \mathrm{~Hz}$ was used. Data from 6 gait cycle was analysed using a: Gait speed $(\mathrm{m} / \mathrm{s})$, Cycle time $(\mathrm{s})$, Stance time (s), Step time (s), Step cadence (s), Stride cadence, Double limb support (s), Double/single limb support(s), Step length, Stride length. Sixteen reflective markers were placed on specific anatomical landmarks in order to obtain the joint segments for hip, knee, and ankle for capturing joint motion. The marker protocol was based on the Plug-in-gait marker set. Data were collected in two times, pre (t0) and post (t1) a10 week intervention of Dance or Physical activity, in order to identify the gait parameters variations. The statistical analysis was performed using SPSS (ver.24.0), gait parameters were compared using repeatedmeasures ANOVA. Statistical significance was accepted for values of $p<.05$.

Participants were randomly assigned to Dance (Dg) or Physical activity (PAg) groups. Participation in the activities lasted 10 weeks, in which each group participated in two weekly training sessions lasting 40 minutes. Each session was organized as follows: 10 minutes of warm-up, 30 minutes of activity (Dance or aerobic exercise in circuit and in pairs), 10 minutes of cooling down and return in terms of activities feeling.

In detail, the 30 minutes of Dance activities included both exercises for two Argentine Tango figures, the "Caminada" and the "Salida Basica", and general rhythm exercises, alone or in pairs; all exercises involved movements of the whole body. The 30 minutes of physical activity included both aerobic exercises in a 5station circuit, in which moments of activity were interspersed with moments of very short rest with a timing of 2 minutes/30 seconds and walking and gait exercises in pairs; each exercise involved the movements of only one part of the body.

All the training sessions, both for the $\mathrm{Dg}$ and for the PAg, included a moment of conclusion with a verbal comparison with the trainers both on the description of the perception of the body and on the feeling of fatigue or post-activity physical stress. The didactic approach used by the trainers was typical of the disciplines taught: for Dance, a metacognitive approach aimed at directing the learning processes by making people aware of the way in which they face motor tasks; for physical activity, a prescriptive approach oriented towards the mere execution of the task.

\section{RESULTS}

Our preliminary results showed that at to the two groups did not differ for all parameters. At t1 a significant improvement in gait parameters was observed in $\mathrm{Dg}$ group. Within $\mathrm{Dg}$, post hoc comparison between $\mathrm{t} 0$ and 
t1 showed a significant effect for several gait parameters (see Table 2). Within PAg, no significant differences were found in all examined parameters (data not shown).

Table 2. Gait analysis data for $\mathrm{Dg}($ mean $\pm \mathrm{SD})$

\begin{tabular}{lcccccc}
\hline Gait & \multirow{2}{*}{$\begin{array}{l}\text { Parameters } \\
\text { Pare-intervention (to) }\end{array}$} & Post-intervention (t1) & \multicolumn{2}{c}{ Time effect } & \multicolumn{2}{c}{ Group effect (t1) } \\
\hline & & & $\mathrm{F}$ & $p$ & $\mathrm{~F}$ & $p$ \\
Gait speed (m/s) & $0.826 \pm 0.120$ & $0.966 \pm 0.156^{*}$ & 23.396 & .001 & 4.684 & .031 \\
Cycle time (s) & $1.178 \pm 0.070$ & $1.114 \pm 0.094$ & 4.392 & .097 & 2.171 & .201 \\
Stance time (s) & $0.749 \pm 0.062$ & $0.710 \pm 0.086^{*}$ & 5.978 & .013 & 9.894 & .003 \\
Step time (s) & $0.575 \pm 0.027$ & $0.495 \pm 0.037^{*}$ & 4.388 & .034 & 1.465 & .402 \\
Step cadence (s) & $103.134 \pm 4.02$ & $106.483 \pm 5.969^{*}$ & 5.435 & .030 & 1.344 & .378 \\
Stride cadence & $52.374 \pm 2.146$ & $54.105 \pm 3.149^{*}$ & 5.129 & .021 & 1.354 & .456 \\
Double limb & $0.278 \pm 0.045$ & $0.202 \pm 0.098^{*}$ & 5.828 & .016 & 40.782 & .001 \\
support (s) & & & & & & \\
Double/single & $0.389 \pm 0.043$ & $0.338 \pm 0.073^{*}$ & 8.748 & .002 & 40.989 & .001 \\
limb support(s) & $0.567 \pm 0.08$ & $0.633 \pm 0.073^{*}$ & 22.511 & .001 & 0.098 & .971 \\
Step length & $1.055 \pm 0.140$ & $1.148 \pm 0.149^{*}$ & 9.998 & .003 & 0.010 & .877 \\
Stride length &
\end{tabular}

Note: Pre-intervention to: baseline evaluation; Post-intervention t1: end of activity at 10 weeks; ': statistically significant as compared to baseline evaluation (pre-test); SD: Standard Deviation; s: seconds; m: meters.

\section{DISCUSSION AND CONCLUSION}

This preliminary study aimed at understanding how different physical activities, of similar metabolic commitment, could affect gait parameters in the elderly. The use of an optoelectronic motion analysis system, considered the most useful tool for measuring motor parameters in a laboratory setting, has allowed us to objectively evaluate how much two different aerobic physical activity interventions, could affect the gait cycle parameters in elderly people not accustomed to motor practice. The interventions consisted in Dance and in an aerobic circuit training, two types of activities widely used as primary preventive strategies for the healthy elderly falls. The idea was born from the ever-increasing demand for physical activity protocols for the elderly and, conversely, from the ever-increasing mix of proposed activities. Several studies have investigated different proposals for physical activities for the elderly but only a few have a rigorous approach to measuring motor performance in the pre- and post-intervention. From the preliminary results obtained, statistically significant improvements are evident in the group that participated in the Dance activities, both in the temporal comparison and in the comparison between groups. In detail, in the $\mathrm{Dg}$ the time effect leads to statistically significant results for all the gait parameters, with the exception of the cycle time. This data could be explained that the subjects were asked to walk at their most comfortable pace. Furthermore, the group effect leads to statistically significant results for gait speed, stance time, double and single limb support, all parameters that have a close relationship with the stability of the gait.

By these data we conclude that, compared to an aerobic physical activity intervention, where the execution of the motor gesture is segmental and stereotyped, a Dance intervention is more effective in improving the organization of the gait. The improvement of the observed gait parameters also allows us to say that this also results in a reduction in the risk of falls. Our study, albeit on a small sample, has shown that aerobic physical activity consisting of Dance exercises can be an affective proposal in preventive falls in the elderly, but its effectiveness is also strictly dependent on the level of the didactic approach. The latter must be structured 
and organized in such a way as to affect the subject's cognitive organization and consequently his motor organization. Only in this case does physical activity become a valid pedagogical-educational strategy useful for modifying motor behaviour in the long term. To our knowledge, to date, no study has investigated, using an optoelectronic system of motion analysis, how effective Dance is on changing the gait parameters in the elderly, even by dealing with the educational level of motor intervention. Further studies are necessary in this sense, with a higher sample size and a long-term monitoring of the motor phenomenon, even after the end of the activities.

\section{AUTHOR CONTRIBUTION}

Valeria Agosti: Conception and design, Analysis and interpretation of data, Drafting and revising the manuscript. Silvia Coppola: Acquisition of data, drafting the manuscript. Rodolfo Vastola: Revising the manuscript critically for important intellectual content, Approval of the version of the manuscript to be published.

\section{REFERENCES}

Brawley, L. R., Rejeski, W. J., \& King, A. C. (2003). Promoting physical activity for older adults: the challenges for changing behavior. American journal of preventive medicine, 25(3), 172-183. https://doi.org/10.1016/s0749-3797(03)00182-x

Cruz-Jimenez, M. (2017). Normal changes in gait and mobility problems in the elderly. Physical Medicine and Rehabilitation Clinics, 28(4), 713-725. https://doi.org/10.1016/j.pmr.2017.06.005

de Labra, C., Guimaraes-Pinheiro, C., Maseda, A., Lorenzo, T., \& Millán-Calenti, J. C. (2015). Effects of physical exercise interventions in frail older adults: a systematic review of randomized controlled trials. BMC geriatrics, 15(1), 154. https://doi.org/10.1186/s12877-015-0155-4

Dhami, P., Moreno, S., \& DeSouza, J. F. (2015). New framework for rehabilitation-fusion of cognitive and physical rehabilitation: the hope for dancing. Frontiers in psychology, 5, 1478. https://doi.org/10.3389/fpsyg.2014.01478

Faber, M. J., Bosscher, R. J., Paw, M. J. C. A., \& van Wieringen, P. C. (2006). Effects of exercise programs on falls and mobility in frail and pre-frail older adults: a multicenter randomized controlled trial. Archives of physical medicine and rehabilitation, 87(7), 885-896. https://doi.org/10.1016/i.apmr.2006.04.005

Hui, E. K. H., \& Rubenstein, L. Z. (2006). Promoting physical activity and exercise in older adults. Journal of the American Medical Directors Association, 7(5), 310-314. https://doi.org/10.1016/j.jamda.2006.03.006

Kshtriya, S., Barnstaple, R., Rabinovich, D. B., \& DeSouza, J. F. (2015). Dance and aging: a critical review of findings in neuroscience. American Journal of Dance Therapy, 37(2), 81-112. https://doi.org/10.1007/s10465-015-9196-7

Mattle, M., Chocano-Bedoya, P. O., Fischbacher, M., Meyer, U., Abderhalden, L. A., Lang, W., Mansky, R., Kressig, R. W., Steurer, J., Orav, E. J., \& Bischoff-Ferrari, H. A. (2020). Association of DanceBased Mind-Motor Activities with Falls and Physical Function among Healthy Older Adults: A Systematic Review and Meta-analysis. JAMA network open, 3(9), e2017688. https://doi.org/10.1001/jamanetworkopen.2020.17688

Rodrigues-Krause, J., Krause, M., \& Reischak-Oliveira, A. (2019). Dancing for Healthy Aging: Functional and Metabolic Perspectives. Alternative Therapies in Health \& Medicine, 25(1).

Stein, M. (2017). Allgemeine Pädagogik: Mit 14 Abb. und 25 Tabellen, mit 56 Übungsaufgaben (Vol. 3215). UTB. 
Veronese, N., Maggi, S., Schofield, P., \& Stubbs, B. (2017). Dance movement therapy and falls prevention. Maturitas, 102, 1-5. https://doi.org/10.1016/j.maturitas.2017.05.004 